\title{
Psoriasin (S100A7) promotes stress-induced angiogenesis
}

\author{
J. Vegfors, ${ }^{1}$ A.-K. Ekman, ${ }^{1}$ S.W. Stoll, ${ }^{2}$ C. Bivik Eding ${ }^{1}$ and C. Enerbäck ${ }^{1}$ \\ ${ }^{1}$ Department of Clinical and Experimental Medicine, Ingrid Asp Psoriasis Research Center, Linköping University, Linköping, Sweden \\ ${ }^{2}$ Department of Dermatology, University of Michigan, Ann Arbor, MI, U.S.A.
}

Linked Comment: Mishra et al. Br J Dermatol 2016; 175:1141-1142.

\section{Correspondence \\ Charlotta Enerbäck. \\ E-mail: charlotta.enerback@liu.se \\ Accepted for publication \\ 30 April 2016 \\ Funding sources \\ This research was funded by the Ingrid Asp Foundation, the Welander Foundation, the Swedish Psoriasis Association and the Medical Research Council.}

\section{Conflicts of interests}

None declared.

DOI $10.1111 /$ bjd. 14718

\section{Summary}

Background Vascular modifications occur early in the development of psoriasis, and angiogenesis is one of the key features in the pathogenesis of the disease.

Objectives To identify the role of the S100 protein psoriasin in psoriasis-associated angiogenesis.

Methods The role of psoriasin in mediating angiogenesis was investigated by silencing psoriasin with small interfering RNA (siRNA) and measuring psoriasisassociated angiogenic factors in human epidermal keratinocytes. The secretion of psoriasin and the effect of psoriasin on general regulators of angiogenesis in keratinocytes, and on endothelial cell migration, proliferation, tube formation and production of angiogenic mediators, was evaluated.

Results Reactive oxygen species (ROS) and hypoxia induced the expression of psoriasin. Downregulation of psoriasin in keratinocytes using siRNA altered the ROSinduced expression of the psoriasis-associated angiogenic factors vascular endothelial growth factor (VEGF), heparin-binding epidermal growth factor-like growth factor, matrix metalloproteinase 1 and thrombospondin 1. Overexpression of psoriasin altered several regulators of angiogenesis and led to the secretion of psoriasin. Treatment with extracellular psoriasin induced proliferation, migration and tube formation in dermal-derived endothelial cells to a similar extent as VEGF and interleukin-17, and induced the expression and release of proangiogenic mediators. These effects were suggested to be mediated by the PI3K and nuclear factor kappa B pathways.

Conclusions These findings suggest that psoriasin expression is promoted by oxidative stress in keratinocytes and amplifies the ROS-induced expression of angiogenic factors relevant to psoriasis. Moreover, extracellularly secreted psoriasin may act on dermal endothelial cells to contribute to key features angiogenesis.

\section{What's already known about this topic?}

- Psoriasis is characterized by extensive new blood vessel formation.

- Early microvascular modifications in the psoriatic lesion have received limited attention in psoriasis pathogenesis.

\section{What does this study add?}

- Psoriasin expression is promoted by reactive oxygen species (ROS) and hypoxia in keratinocytes. It regulates the ROS-induced expression of several psoriasis-associated angiogenic factors, and acts on dermal endothelial cells to promote key features of the angiogenic process.

\section{What is the translational message?}

- Our data raise the possibility that psoriasin may be evaluated as a novel antiangiogenic target in psoriasis. 
Psoriasis is a chronic inflammatory skin disease characterized by an intense hyperproliferation and a disturbed maturation of keratinocytes, an inflammatory dermal infiltrate and the formation of new blood vessels, in particular alterations of postcapillary venules within the rete ridges. ${ }^{1-4}$

Microvascular modifications constitute one of the earliest detectable changes in the development of the psoriatic lesion. ${ }^{5,6}$ These modifications include dilatation, hyperpermeability, tortuosity and elongation of dermal blood vessels and proliferation of endothelial cells. ${ }^{7}$ The vascular alterations in psoriasis promote skin inflammation through the recruitment of leucocytes to lesional skin. ${ }^{8}$ Several proangiogenic factors are upregulated in the psoriatic epidermis. Vascular endothelial growth factor (VEGF), a major epidermis-derived growth factor, is strongly upregulated in psoriatic skin, ${ }^{9}$ and is important in the initiation of the psoriatic phenotype. ${ }^{10}$ Interestingly, increased expression of VEGF is also present in nonlesional skin. ${ }^{11}$ Transgenic mice overexpressing VEGF develop an inflammatory skin disorder resembling psoriasis, with an increased density of tortuous capillaries. ${ }^{12}$ Many of the treatments used clinically, including tumour necrosis factor (TNF) $-\alpha$ inhibitors, methotrexate and phototherapy, have antiangiogenic properties. ${ }^{8}$ Despite this, the vascular contribution to the pathogenesis of psoriasis has generally received limited attention. In fact, angiogenesis in psoriasis was recently identified as a 'research gap' in the field of psoriasis. ${ }^{13}$

Psoriasin (S100A7) was originally identified as being highly expressed in psoriatic lesions. ${ }^{14}$ It belongs to the $\mathrm{S} 100$ family of calcium-binding proteins that are involved in several cellular processes, including proliferation, differentiation, invasion and metastasis. ${ }^{15,16}$ An elevated expression of psoriasin has been detected in some carcinomas of epithelial origin, such as squamous cell carcinoma of the skin and bladder, ${ }^{17,18}$ as well as in breast cancer, where its expression has been linked to features of poor prognosis. ${ }^{19,20}$

In the skin, the expression of psoriasin is especially pronounced in psoriasis; however, it is also elevated in other hyperproliferative and inflammatory disorders. ${ }^{21}$ Psoriasin has a distinct antimicrobial effect on Escherichia coli, ${ }^{14,22,23}$ and has been shown to be chemotactic for $\mathrm{CD}^{+} \mathrm{T}$ cells and neutrophils. ${ }^{24}$ Psoriasin is induced by several proinflammatory cytokines present in psoriatic skin, such as interleukin (IL)-1, TNF- $\alpha$, IL- 17 and IL-22. ${ }^{25}$

The high expression of psoriasin in the psoriatic plaque, along with its elevated expression in other conditions characterized by angiogenesis, makes it a relevant protein to study in the context of angiogenesis.

In this study, we aimed to identify the role of psoriasin in psoriasis-associated angiogenesis.

\section{Materials and methods}

\section{Cell culture conditions and treatments}

Neonatal human epidermal keratinocytes (HEKn) were cultured in Epilife medium (Cascade Biologics, Paisley, U.K.) as previously described. ${ }^{26}$ Human embryonic kidney cells (293FT; Invitrogen, Carlsbad, CA, U.S.A.) were grown in Dulbecco's modified Eagle's medium (Gibco, Carlsbad, CA, U.S.A.) supplemented with $10 \%$ fetal bovine serum (Gibco). The immortalized, nontransformed keratinocyte cell line N/ TERT-2G was grown in keratinocyte serum-free medium (Gibco) as previously described. ${ }^{27}$ Human dermal-derived microvascular endothelial cells (HMVEC-d) were cultured in endothelial basal medium supplemented with endothelial cell growth medium-2 BulletKit (Lonza, Walkersville, MD, U.S.A.). Recombinant human psoriasin was purchased from Abnova (Taipei, Taiwan); VEGF was obtained from Invitrogen and the IL-17 from R\&D systems (Minneapolis, MN, U.S.A.). The inhibitors LY294002 and caffeic acid phenethyl ester (CAPE) were from Sigma Aldrich (St Louis, MO, U.S.A.).

\section{Induction of cellular stress}

To study the effect of stress conditions on psoriasin production, HEKn were seeded at a density of 500000 cells/T25 culture flask in complete Epilife medium. Following adherence, the medium was replaced with fresh medium containing 150 or $250 \mu \mathrm{mol} \mathrm{L}{ }^{-1} \mathrm{H}_{2} \mathrm{O}_{2}$ (Sigma Aldrich) for 24-48 h $(\mathrm{n}=4)$, or $500 \mu \mathrm{mol} \mathrm{L}{ }^{-1} \mathrm{CoCl}_{2}$ (Sigma-Aldrich) for $24 \mathrm{~h}$ $(n=4)$. To induce hypoxia $(n=6)$, the adhered cells were given fresh medium and were subsequently moved to a hypoxic chamber with $1 \% \mathrm{O}_{2}$ where they remained for $24 \mathrm{~h}$. Following treatment, cells were harvested and lysed in RLTbuffer (Qiagen, Hilden, Germany) for RNA extraction. Protein quantification was performed nine times in $\mathrm{H}_{2} \mathrm{O}_{2}$-treated cells and seven times in hypoxia-treated cells.

\section{Small interfering RNA}

Psoriasin small interfering RNA and a nontargeting siRNA were purchased from Santa Cruz Biotechnology (Santa Cruz, CA, U.S.A.). HEKn were transfected with siRNA transfection reagent and siRNA in Opti-MEM (Gibco). Cells were stimulated with $\mathrm{H}_{2} \mathrm{O}_{2} 24 \mathrm{~h}$ after transfection. The downregulation of psoriasin was confirmed by quantitative real-time polymerase chain reaction ( $\mathrm{qPCR}$ ). A total of six independent experiments were performed.

\section{RNA extraction, cDNA synthesis and quantitative real- time polymerase chain reaction}

RNA was prepared using the RNeasy mini kit (Qiagen) and cDNA was synthesized using the Maxima First Strand cDNA Synthesis Kit (Fermentas, Vilnius, Lithuania). mRNA expression was analysed with qPCR on a real-time $7500 \mathrm{HT}$ sequence detection system with SYBR green detection (Applied Biosystems, Foster City, CA, U.S.A.). The primers were as previously described, ${ }^{28-32}$ or were designed using Primer Express Software v3.0 (Applied Biosystems). All primer sequences are listed in Table S1 (see Supporting Information). GAPDH or RPLP0 were used as housekeeping genes. The relative 
expression of the target genes to untreated controls was determined using the comparative $C_{\mathrm{t}}$ method.

\section{Cell proliferation and viability}

Human dermal-derived microvascular endothelial cells were seeded at 7500 cells per well in a 96-well plate and were allowed to adhere before treatment. After adherence, the cell culture medium was replaced with basal medium containing either psoriasin $\left(0 \cdot 15 \mu \mathrm{g} \mathrm{mL}^{-1}\right.$ or $\left.1 \mu \mathrm{g} \mathrm{mL}^{-1}\right)$ or VEGF $\left(10 \mathrm{ng} \mathrm{mL} \mathrm{mL}^{-1}\right)$. Cells were allowed to proliferate for $24 \mathrm{~h}$ and proliferation and viability were determined using a CellTiter $96{ }^{\circledR}$ AQueous One Solution Cell proliferation assay [3-(4,5dimethylthiazol-2-yl)-5-(3-carboxymethoxyphenyl)-2-(4-sulfophenyl)-2H-tetrazolium (MTS) $(\mathrm{n}=5)$; Promega, Madison, WI, U.S.A.] or PrestoBlue cell viability reagent $(n=4$; Invitrogen), according to the manufacturer's instructions. The results were further verified in 12 -well plates and by counting after 24, 48 and $72 \mathrm{~h}$ of treatment, which revealed a significant increase in proliferation at $24 \mathrm{~h}$ but not at later time points (data not shown). To examine whether VEGF mediated the proliferative response, cells were treated with $0.2 \mu \mathrm{g} \mathrm{mL}^{-1}$ VEGF-neutralizing antibody (R\&D Systems), followed by treatment with psoriasin or VEGF for $24 \mathrm{~h}$. The absorbance was recorded at the recommended wavelengths using the VersaMax Microplate Reader (Molecular Devices, Sunnyvale, CA, U.S.A.). The relative absorbance was calculated for treated cells vs. untreated cells.

\section{Tube formation assay}

The formation of HMVEC-d in capillary-like structures was studied on Geltrex-reduced growth factor basement membrane matrix (Invitrogen), according to the manufacturer's instructions. Briefly, a total of 60000 HMVEC-d cells were seeded onto the matrix and were allowed to adhere. After adherence, cells were treated with psoriasin $\left(0 \cdot 15 \mu \mathrm{g} \mathrm{mL}^{-1}\right.$ or $1 \mu \mathrm{g} \mathrm{mL}^{-1}$; Abnova) or VEGF (10 $\left.\mathrm{ng} \mathrm{mL}^{-1}\right)$. Tube formation was visualized after $18 \mathrm{~h}$ of treatment using an Olympus IX51 inverted microscope and an Olympus DP70 camera (Olympus, Tokyo, Japan). Three independent experiments were performed. Tube formation assays were quantified in ImageJ (National Institutes of Health, Bethesda, MD, U.S.A.) with the macro Angiogenesis Analyzer for ImageJ, detecting the total length, defined by the sum of length of segments, isolated elements and branches. As these experiments consisted of only three repeats, statistical comparison of the quantifications was not deemed meaningful.

\section{Scratch migration assay}

The ability of psoriasin to mediate the migration of HMVEC-d was evaluated in a scratch migration assay. Cells were seeded densely in complete medium in 96-well plates and were allowed to adhere. When the cells had adhered, the confluent HMVEC-d monolayer was scratched with a pipette tip and washed with phosphate-buffered saline to remove debris. The medium was subsequently replaced with basal medium containing psoriasin $\left(0 \cdot 15 \mu \mathrm{g} \mathrm{mL}^{-1}\right.$ or $\left.1 \mu \mathrm{g} \mathrm{mL}^{-1}\right)$ or $\mathrm{IL}-17$ (50 $\mathrm{ng} \mathrm{mL} \mathrm{mL}^{-1}$ ), used as a positive control as it is a key cytokine in psoriasis. To inhibit the PI3K and nuclear factor kappa B (NFKB) pathways, the inhibitors LY204002 $\left(5 \mu \mathrm{mol} \mathrm{L}{ }^{-1}\right)$ and CAPE $\left(50 \mu \mathrm{mol} \mathrm{L}{ }^{-1}\right)$ were applied to the relevant wells $45 \mathrm{~min}$ prior to psoriasin or IL-17 treatment. DMSO treatment was used as diluent control. Cells were allowed to migrate in reduced serum media containing treatment for $18 \mathrm{~h}$. Migration of HMVEC-d was visualized using an Olympus IX51 inverted microscope and an Olympus DP70 camera. A total of three independent experiments were performed. Scratch assays were quantified in ImageJ using the detect edges feature and by measuring the mean intensity in the area of the scratch. As these experiments consisted of only three repeats, statistical comparison of the quantifications was not deemed meaningful.

\section{Directional cloning of the psoriasin sequence}

Total RNA was extracted from homogenized human psoriatic skin biopsies and cDNA synthesis was performed. Psoriasin (NM_002963) was amplified by PCR with gene-specific primers. The forward primer included a short sequence at the $5^{\prime}$ end (CACC) for directional cloning into the pENTR/D-TOPO vector (Invitrogen). After PCR amplification and gel purification, the psoriasin DNA was cloned into pENTR followed by transformation into One Shot TOP10 chemically competent E. coli (Invitrogen). Insert DNA from positive clones was verified by DNA sequencing with M13 forward (5'GTAAAA CGACGGCCAG- $3^{\prime}$ ) and reverse (5'-CAGGAAACAGCTATGAC- $3^{\prime}$ ) primers.

\section{Lentivirus-mediated gene expression}

cDNA encoding the psoriasin gene was cloned into the lentiviral expression vectors pLenti6.3/TO/V5-DEST and used to produce infectious lentivirus particles in 293FT cells, according to the manufacturer's instructions (Invitrogen). Keratinocytes with constitutive psoriasin expression were generated by infection of N/TERTs with the lentivirus constructs followed by selection with $10 \mu \mathrm{g} \mathrm{mL}^{-1}$ blasticidin. N/TERTs were seeded at a density of 5000 cells per $\mathrm{cm}$ and grown to $40 \%$ confluence. The medium was then replaced with new medium that was harvested after 24, 48 and $72 \mathrm{~h}$, and cells were lysed with RLT buffer (Qiagen).

\section{Enzyme-linked immunosorbent assay}

The secreted levels of psoriasin, VEGF, matrix metalloproteinase (MMP)-9 and IL-8 were quantitated using psoriasin/S100A7 (Circulex, Woburn, MA, U.S.A.), VEGF Quantikine (R\&D Systems), MMP9 Quantikine (R\&D Systems) or IL-8 Quantikine (R\&D Systems) ELISA kits, according to the manufacturer's instructions. ELISAs were performed on cell culture supernatant or total protein extract. To obtain total protein, cells were lysed in RIPA buffer (Thermo scientific, Rockford, IL, U.S.A.) and the 
protein level was measured with a Bio-Rad protein assay (Hercules, CA, U.S.A.). The protein was diluted in assay buffer before the ELISA assay, to avoid RIPA interference. The absorbance of the assays was measured on a VersaMax Microplate Reader (Molecular Devices) at the recommended wavelengths.

\section{Gene expression microarray}

RNA (100 ng) extracted from three psoriasin-expressing or control N/TERTs $(n=3)$ after $24 \mathrm{~h}$ of culture was used to generate hybridization probes using the GeneChip ${ }^{\circledR}$ WT PLUS Reagent kit (Affymetrix, Santa Clara, CA, U.S.A.), according to the manufacturer's instructions. Hybridization to GeneChip ${ }^{\circledR}$ human transcriptome 2.0 arrays was performed in a GeneChip ${ }^{\circledR}$ hybridization oven, followed by washing and staining in a GeneChip ${ }^{\circledR}$ Fluidics Station 450 and scanning in a GeneChip $^{\circledR}$ Scanner $30007 \mathrm{G}$ system. Expression Console software (version 1.2.1) was used to verify the quality controls. CELfiles generated by the Affymetrix AGCC program were analysed with GeneSpring GX software (version 13.0; Agilent Technologies, Santa Clara, CA, U.S.A.). A standard robust multiarray average normalization and background correction was performed. The probe sets were filtered on expression with a twentieth percentile cut-off. The fold change was computed by the GeneSpring software as a ratio between the psoriasinoverexpressing cells against the control cells. On the probe sets that remained after the filtering, a 1.5-fold change was applied as a cut-off for up- and downregulated genes. Heat maps of the differentially regulated genes were constructed based on normalized, filtered and $\log _{2}$-transformed data with Euclidean similarity measure. Probe sets with a fold change $\geq 1.5$ were mapped on the gene ontology (GO) function, selecting for 'regulators of angiogenesis' and 'positive regulators of angiogenesis' as biological functions.

\section{Statistical analysis}

Statistical analysis was performed in GraphPad Prism 6 (GraphPad Software, La Jolla, CA, U.S.A.), using a paired or unpaired Student's t-test and Wilcoxon's signed rank test. A value of $\mathrm{P} \leq 0.05$ was considered statistically significant. The results are presented as mean $\pm \mathrm{SD}$.

\section{Results}

\section{Psoriasin expression is induced by cellular stress in keratinocytes}

Proangiogenic factors are triggered by stress signals, such as oxidative stress and hypoxia. In psoriasis, the increased production of reactive oxygen species (ROS) overrides the effect of endogenous antioxidants. ${ }^{33}$ Treatment with $\mathrm{H}_{2} \mathrm{O}_{2}$ induced the expression of psoriasin in keratinocytes (Fig. 1a, b), with the highest induction seen upon treatment with $250 \mu \mathrm{mol} \mathrm{L}{ }^{-1} \mathrm{H}_{2} \mathrm{O}_{2}$ after $48 \mathrm{~h}$. As expected, $\mathrm{H}_{2} \mathrm{O}_{2}$ also led to the expression of VEGF (Figs $2 \mathrm{~b}$ and 1c).
Hypoxia has been shown to induce the production of $\mathrm{ROS}^{34}$ and a hypoxic state has been suggested in the psoriatic plaque. ${ }^{35}$ We therefore cultured HEKn in a hypoxic chamber with $1 \% \mathrm{O}_{2}$ for $24 \mathrm{~h}$. This culture significantly induced the expression of psoriasin (Fig. 1d,f), as well as the expression of VEGF, which functioned as a positive control (Fig. 1e,g). Similarly, the hypoxia-mimicking agent, $\mathrm{CoCl}_{2}$, induced both psoriasin (Fig. 1h) and VEGF expression (Fig. 1i). Our data demonstrate that the oxidative stress factors $\mathrm{H}_{2} \mathrm{O}_{2}$, hypoxia and $\mathrm{CoCl}_{2}$ all induce psoriasin expression in keratinocytes.

\section{Psoriasin mediates $\mathrm{H}_{2} \mathrm{O}_{2}$-induced expression of psoriasis- associated angiogenic factors in keratinocytes}

To determine whether psoriasis-associated factors of angiogenesis are affected by the expression of psoriasin, the levels of these factors were measured after treating keratinocytes with $\mathrm{H}_{2} \mathrm{O}_{2}$. In addition to giving rise to induced psoriasin expression (Fig. 2a), this treatment also increased the levels of the proangiogenic factors VEGF (Fig. 2b), heparin-binding epidermal growth factor-like growth factor (HB-EGF; Fig. 2c), MMP-1 (Fig. 2d), MMP-9 (Fig. 2e) and IL-8 (Fig. 2f), whereas the expression of the antiangiogenic factor thrombospondin (THBS)-1 (Fig. 2g) was reduced. To investigate the specific effect of psoriasin in the $\mathrm{H}_{2} \mathrm{O}_{2}$-promoted induction of these factors, we downregulated the endogenous expression of psoriasin using siRNA. The efficient downregulation of psoriasin was confirmed by qPCR (Fig. 2a). The expression of the proangiogenic factors VEGF (Fig. 2b), HB-EGF (Fig. 2c) and MMP-1 (Fig. 2d) was found to be significantly decreased in cells with downregulated psoriasin expression, suggesting that psoriasin participates in their regulation. The expression of MMP-9 (Fig. 2e) and IL-8 (Fig. 2f) was decreased without reaching statistical significance. Furthermore, the expression of the antiangiogenic factor THBS-1 was significantly increased in cells with downregulated psoriasin expression. These results suggest that psoriasin amplifies the angiogenic response to $\mathrm{H}_{2} \mathrm{O}_{2}$ in keratinocytes.

\section{Psoriasin does not influence angiogenic markers by upregulating vascular endothelial growth factor receptors}

We speculated that psoriasin might influence these angiogenic factors through the induction of the VEGF receptors R1 and R2, as many of these markers are targets of VEGF. Treatment with extracellular psoriasin did not induce the mRNA expression of VEGFR1 and VEGFR2 in keratinocytes, nor did the treatment synergize with VEGF to upregulate these receptors (data not shown).

\section{Overexpression of psoriasin leads to secretion of psoriasin into the extracellular space and altered expression of general regulators of angiogenesis}

To further determine the effect of psoriasin on angiogenesis and the potential of the protein to influence surrounding cells, 
Fig 1. Psoriasin is induced by angiogenesispromoting stress stimuli in keratinocytes. Neonatal human epidermal keratinocytes (HEKn) were either treated with $\mathrm{H}_{2} \mathrm{O}_{2}(150-$ $250 \mu \mathrm{mol} \mathrm{L}{ }^{-1}$ ) for $48 \mathrm{~h}$, cultured in hypoxia or treated with the hypoxia-mimicking agent $\mathrm{CoCl}_{2}\left(500 \mu \mathrm{mol} \mathrm{L}{ }^{-1}\right)$, for $24 \mathrm{~h}$. The expression of psoriasin and vascular endothelial growth factor (VEGF) was analysed using quantitative real-time polymerase chain reaction. (a) $\mathrm{H}_{2} \mathrm{O}_{2}$ treatment increased psoriasin mRNA expression compared with untreated cells $(n=4)$, as well as the expression of both (b) psoriasin $(n=9)$ and (c) VEGF protein $(\mathrm{n}=9)$. Furthermore, culture in hypoxia increased psoriasin at both the (d) mRNA $(\mathrm{n}=6)$ and $(\mathrm{f})$ protein $(\mathrm{n}=7)$ level. Hypoxia also successfully induced the (e) mRNA $(\mathrm{n}=6)$ and $(\mathrm{g})$ protein $(\mathrm{n}=7)$ of the positive control, VEGF. The treatment of HEKn with $\mathrm{CoCl}_{2}$ induced the expression of (h) psoriasin and the expression of (i) VEGF $(n=4)$. Gene expression levels are shown as the relative fold induction vs. untreated controls. (a) GAPDH or (d, e, h, i) RPLP0 were used as reference genes. Psoriasin protein expression is measured as ng per mg of total protein and VEGF as pg per mg of total protein. Data are presented as mean $\pm \mathrm{SD}$. $* \mathrm{P}<0.05$ and $* * \mathrm{P}<0.01 . \mathrm{ns}$, nonsignificant.
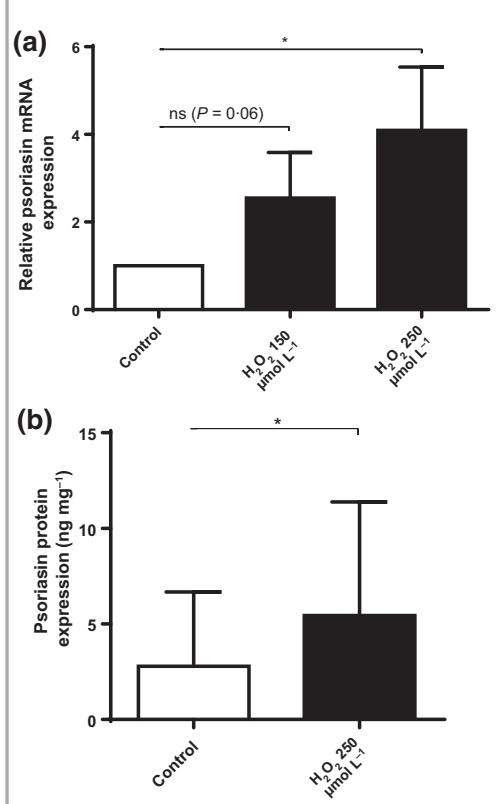

(c)
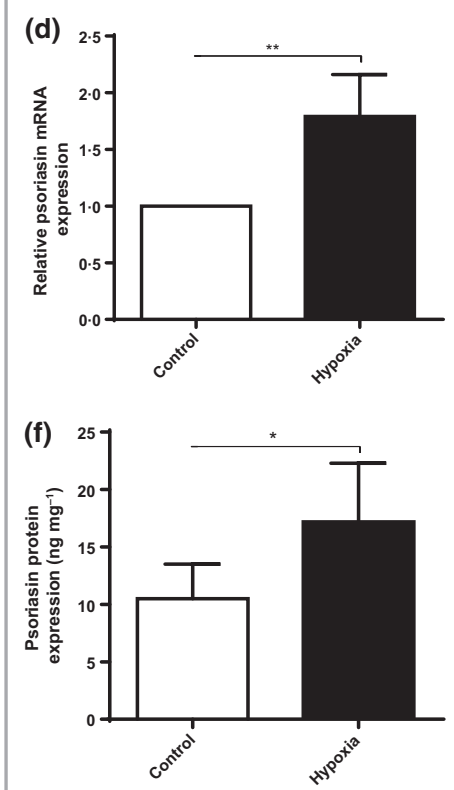

(h)

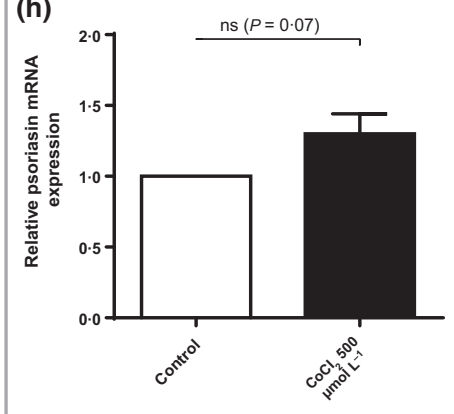

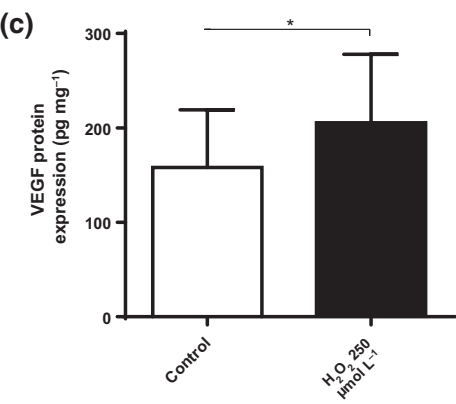
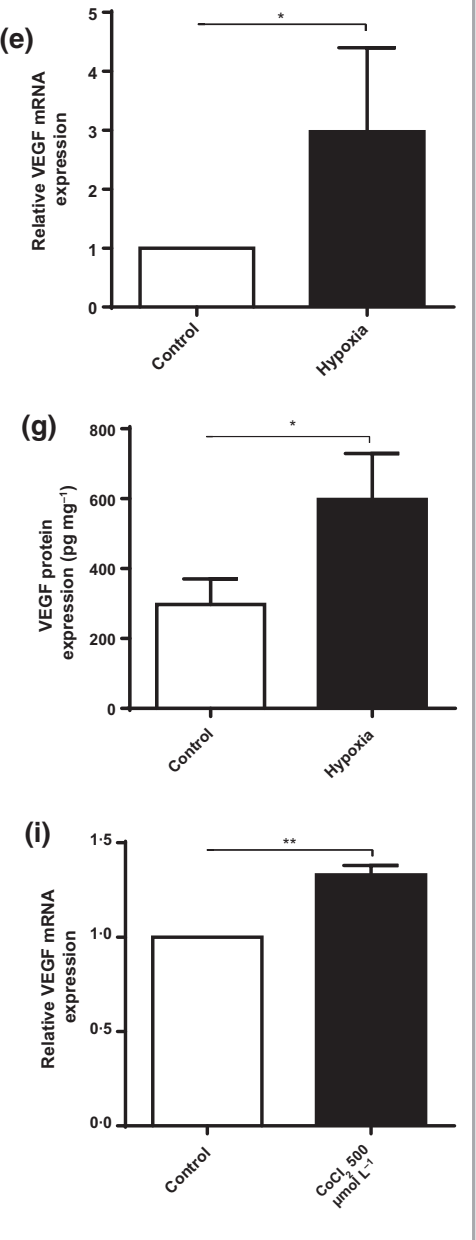

we generated keratinocytes with constitutively overexpressed psoriasin. The efficient upregulation of psoriasin expression was confirmed with qPCR (data not shown) and with a psoriasin ELISA, which revealed a pronounced psoriasin expression at all the investigated time points (Fig. 2h). These results demonstrate not only the successful overexpression of psoriasin in the generated cells, but also that psoriasin protein is highly secreted into the extracellular environment. The 


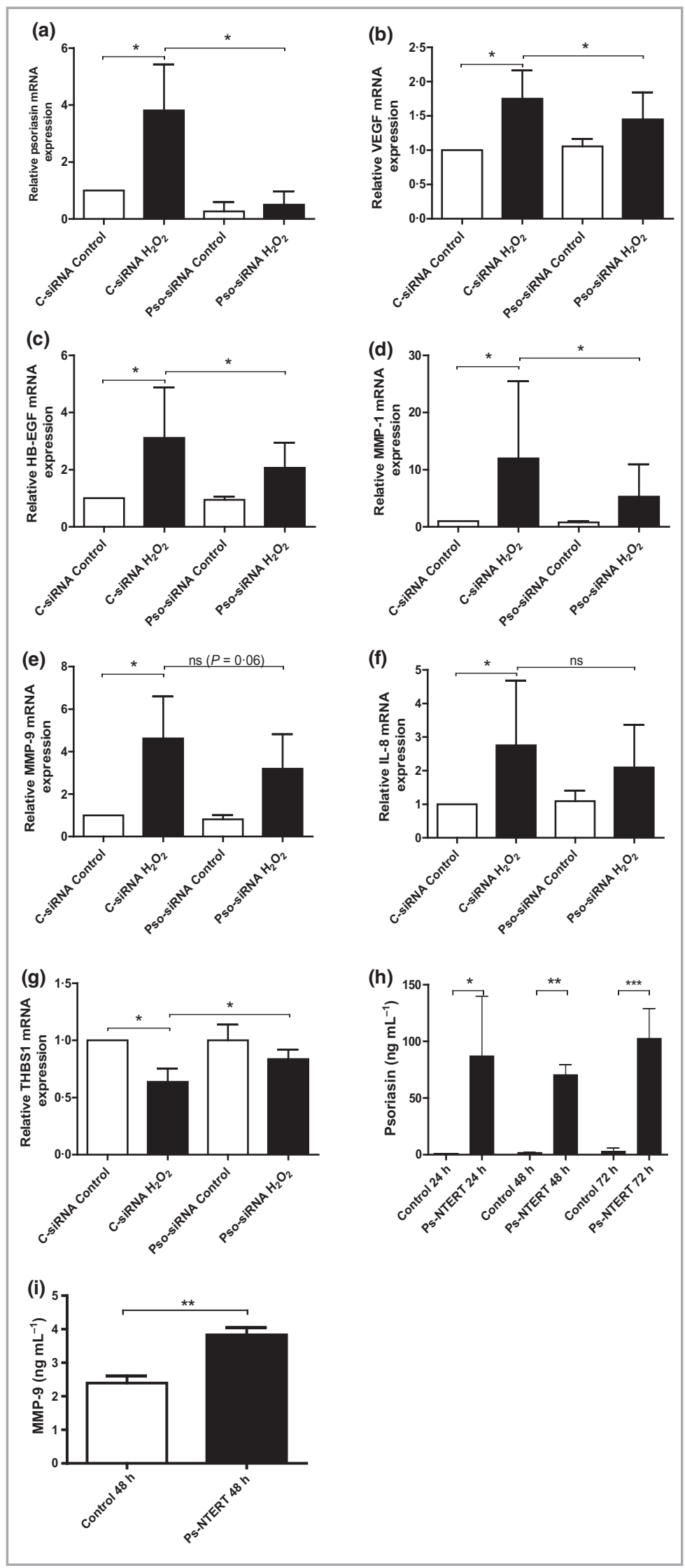

Fig 2. The downregulation of psoriasin expression in $\mathrm{H}_{2} \mathrm{O}_{2}$-treated keratinocytes regulates the expression of angiogenic factors. Neonatal human epidermal keratinocytes (HEKn) were transfected with small interfering (siRNA) directed against psoriasin (Pso-siRNA) or negative control siRNA (CsiRNA), and were treated with $250 \mu \mathrm{mol} \mathrm{\textrm {L } ^ { - 1 }}$ $\mathrm{H}_{2} \mathrm{O}_{2}$ for 48 h. Expression was analysed by quantitative polymerase chain reaction. (a) The downregulation of psoriasin was confirmed in $\mathrm{H}_{2} \mathrm{O}_{2}$-treated HEKn. The downregulation of psoriasin suppressed the $\mathrm{H}_{2} \mathrm{O}_{2}$-induced mRNA expression of (b) vascular endothelial growth factor (VEGF), (c) heparin-binding epidermal growth factor-like growth factor (HB-EGF), (d) matrix metalloproteinase (MMP)-1, (e) MMP-9 and (f) IL-8, and (g) increased the mRNA expression of thrombospondin (THBS)- 1 . mRNA expression is normalized to GAPDH. The results are shown as the relative expression of treated cells in relation to untreated cells $(n=6)$. To study the effect of psoriasin on general regulators of angiogenesis, keratinocytes overexpressing psoriasin were generated. The generated keratinocytes (ps-NTERT) secreted markedly higher levels of (h) psoriasin than the control cells at all investigated time points $(\mathrm{n}=3)$ and (i) a higher level of MMP-9 (ng mL ${ }^{-1}$ ) after $48 \mathrm{~h}(\mathrm{n}=3)$. Data are expressed as mean $\pm \mathrm{SD}$. ${ }^{*} \mathrm{P}<0.05,{ }^{*} \mathrm{P}<0.01$, $* * * \mathrm{P}<0.001 . \mathrm{ns}$, nonsignificant. 
overexpression of psoriasin was accompanied by an upregulation of MMP-9 (Fig. 2i).

To determine the effect of this overexpression on angiogenic factors, we performed a microarray expression analysis where we searched for general regulators of angiogenesis. A total of 56372 probe sets were above the twentieth percentile of expression on all arrays and were further analysed. Of these, 283 probe sets had a fold change of $\geq 1.5$ in the comparison between cells with upregulated psoriasin and control cells.

The overexpression of psoriasin induced the upregulation of several factors which by the GO pathways were associated with the regulation of angiogenesis, including the complement component 3, IL-1 $\alpha$, IL-1 $\beta$, MMP-9, coagulation factor 3 , THBS-1 and TNF- $\alpha$-induced protein 3 . The GO pathways also identified the downregulated gene KRT1 as a regulator of angiogenesis (Fig. S1; see Supporting Information).

Furthermore, several factors that have been suggested to regulate angiogenesis were altered, including MMP-10, plasminogen activator urokinase and transforming growth factorB2 (Table S2; see Supporting Information), which suggests that psoriasin affects a broad range of general regulators of angiogenesis.

\section{Psoriasin induces cell proliferation, migration and capillary-like tube formation in dermal endothelial cells}

Key steps in the angiogenic process involve endothelial cell proliferation, migration through the extracellular matrix and the differentiation of endothelial cells into a network of tubes.

In order to study the effect of secreted psoriasin on these steps, HMVEC-d were treated with psoriasin and investigated using an MTS and a Presto Blue viability assay to determine proliferation, a scratch assay to study migration and a tube network formation assay to evaluate angiogenic capacity.

Extracellular psoriasin induced the proliferation of the endothelial cells, as determined by the MTS assay (Fig. 3a) and the Presto Blue viability assay (Fig. 3b). This effect was found to be independent of VEGF as VEGF-neutralizing antibody did not abolish the proliferation (Fig. 3c). Psoriasin also caused a pronounced cellular migration of HMVEC-d (Fig. 3d), comparable with that induced by the positive control, VEGF. Furthermore, psoriasin triggered the formation of tube networks and generated more cell connections on Geltrex matrix than the basal medium control (Fig. 3e). In conclusion, our results demonstrate that extracellular psoriasin increases the angiogenic properties of dermal endothelial cells, to an extent comparable with that of extracellular VEGF.

\section{Psoriasin-induced dermal-derived endothelial cell migration is mediated through the $\mathrm{Pl}_{3} \mathrm{~K}$ and nuclear factor kappa B pathways}

Vascular endothelial growth factor and IL-17 activate the PI3K and NFKB signalling pathways in endothelial cells and regulate downstream target molecules involved in blood vessel growth and homeostasis. ${ }^{36,37}$ To investigate whether these signalling pathways also underlie psoriasin-induced HMVEC-d migration, we examined the effect of inhibitors of PI3K (LY294002) or NFKB (CAPE) following treatment with psoriasin or IL-17. IL17 was shown to stimulate HMVEC-d migration and was also used as the positive control (Fig. 3f). The promoting effect of psoriasin on HMVEC-d migration was effectively blocked by the inhibition of the PI3K (Fig. 3g) and NFKB (Fig. 3h) pathways. LY294002 and CAPE alone had no effect on HMVEC-d migration, nor did dimethyl sulfoxide, used as a diluent control.

\section{Psoriasin induces the expression of angiogenic factors in dermal endothelial cells}

To acquire further insights into the mechanisms by which psoriasin increases angiogenesis, we analysed intracellular angiogenic factors in HMVEC-d cells in response to extracellular psoriasin. We demonstrated a significant increase in the mRNA expression of the proangiogenic factors VEGF (Fig. 4a) and IL-8 (Fig. 4b). Although not statistically significant, we observed an increase in MMP-1 (Fig. 4c) and IL-6 (Fig. 4d) expression. The expression of the antiangiogenic factor THBS1 was found to be reduced by treatment with psoriasin (Fig. 4e). In addition, treatment with psoriasin upregulated the expression of the psoriasin receptor RAGE (receptor for advanced glycation endproducts), suggesting that psoriasin exposure might further potentiate cell reactivity to the protein (Fig. 4f).

Furthermore, IL-8 protein secretion from the HMVEC-d cells was increased after psoriasin treatment, as demonstrated by ELISA (Fig. 4g). Extracellular psoriasin thus induces the expression and release of proangiogenic cytokines in dermal endothelial cells.

\section{Discussion}

Reactive oxygen species play an important role in signal transduction and induce the transcription of cytokines, chemokines and growth factors, which participate in many biological processes, including angiogenesis. ${ }^{38}$ We have previously shown that psoriasin is induced by $\mathrm{H}_{2} \mathrm{O}_{2}$ in mammary epithelial cells and induces human umbilical vein endothelial cell proliferation. $^{32,39}$ We have also demonstrated that the downregulation of psoriasin in a breast cancer cell line with a high endogenous expression of psoriasin led to decreased tumour growth in vivo. ${ }^{28}$ In line with these findings, we also found the upregulation of MMP-13 and the downregulation of VEGF in cells with reduced psoriasin levels. Moreover, we observed a significant positive correlation between psoriasin expression and blood vessel density, determined by the immunohistochemical analysis of psoriasin and CD31, an endothelial cell-specific marker. $^{28}$

In this study, we detected the upregulation of psoriasin by cellular stress $\left(\mathrm{H}_{2} \mathrm{O}_{2}\right.$ and hypoxia $)$ in keratinocytes. 


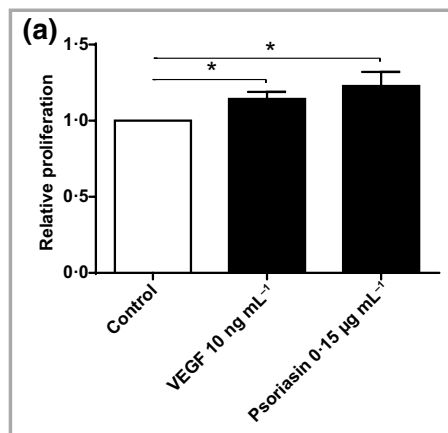

(d)

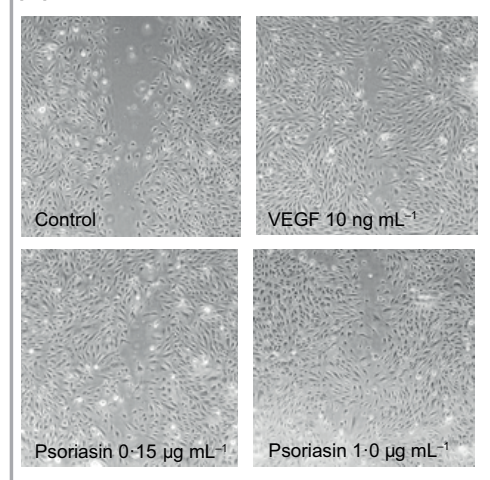

(e)

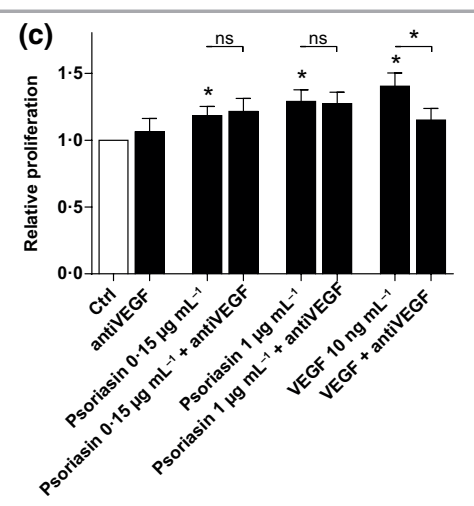

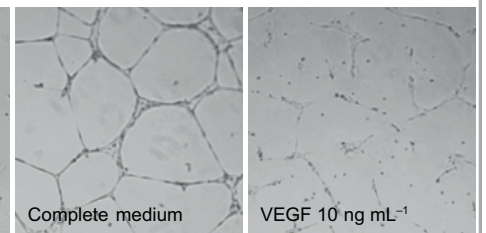
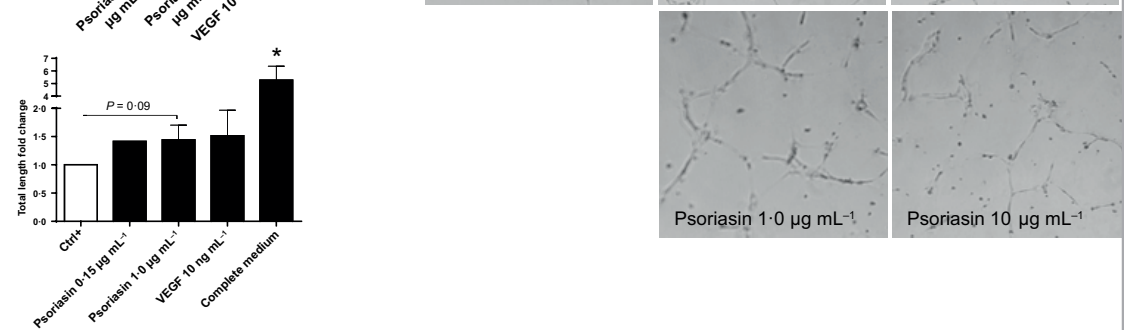

(f)

(g)
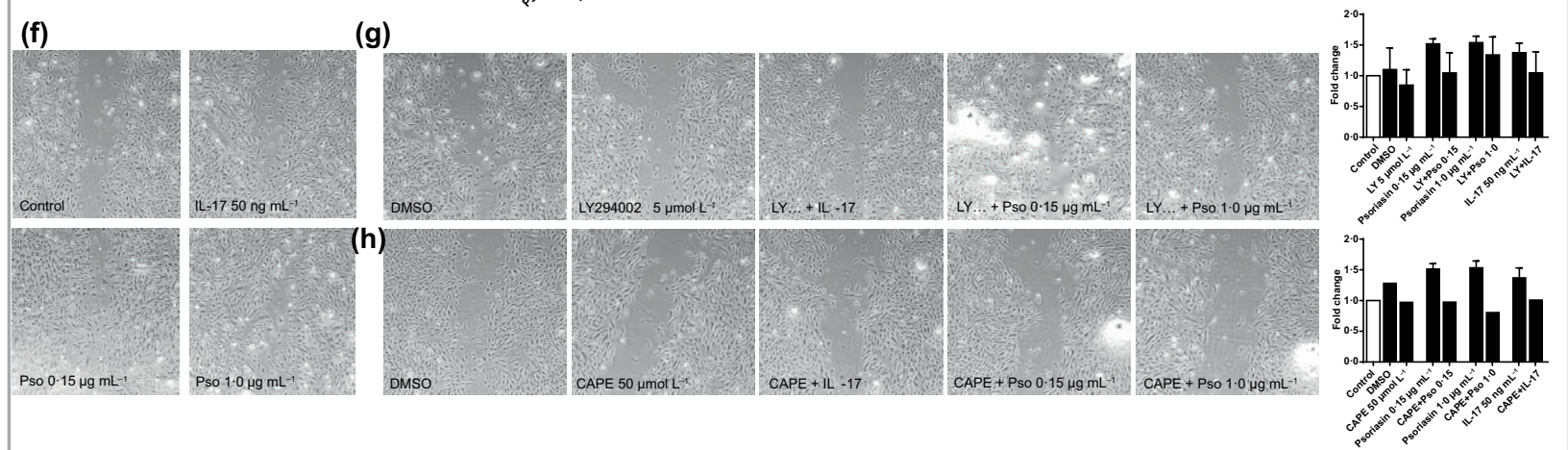

Fig 3. Psoriasin induces cell proliferation, migration and the capillary-like tube formation of dermal-derived endothelial cells. Human dermalderived microvascular endothelial cells (HMVEC-d) were treated with psoriasin $\left(0 \cdot 15-1.00 \mu \mathrm{g} \mathrm{mL}^{-1}\right.$ ) or vascular endothelial growth factor (VEGF; $\left.10 \mathrm{ng} \mathrm{mL}{ }^{-1}\right)$ for $24 \mathrm{~h}$. The quantity of viable cells in proliferation was measured by (a) the 3-(4,5-dimethylthiazol-2-yl)-5-(3carboxymethoxyphenyl)-2-(4-sulfophenyl)-2H-tetrazolium (MTS) assay $(n=5)$ or $(b)$ the PrestoBlue cell viability reagent $(n=4)$. (c) Treatment with VEGF-neutralizing antibody $\left(0 \cdot 2 \mu \mathrm{g} \mathrm{mL}^{-1}\right)$ did not abolish the psoriasin-induced proliferation, measured by PrestoBlue $(\mathrm{n}=7)$. The results are presented as the relative absorbance of the treated cells in relation to the untreated cells. The bar represents the mean $\pm \mathrm{SD}(* \mathrm{P}<0 \cdot 05, * * \mathrm{P}<0 \cdot 01)$. (d) In a subsequent experiment, HMVEC-d were grown to confluence and scratched, followed by treatment with psoriasin $\left(0 \cdot 15-1 \cdot 0 \mu \mathrm{g} \mathrm{mL}{ }^{-1}\right)$ or VEGF $\left(10 \mathrm{ng} \mathrm{mL}{ }^{-1}\right)(\mathrm{n}=3)$. (e) HMVEC-d cells were also seeded onto Geltrex and treated with psoriasin or VEGF for $18 \mathrm{~h}$ and capillary-like tube formation was photographed $(\mathrm{n}=3)$. Basal medium served as a negative control, while VEGF and complete medium were used as positive controls. To investigate the pathways involved in the psoriasin-induced cell migration, HMVEC-d were grown to confluence, scratched and pretreated with LY204002 $\left(5 \mu \mathrm{mol} \mathrm{L}{ }^{-1}\right)$ or caffeic acid phenethyl ester (CAPE; $50 \mu \mathrm{mol} \mathrm{L}^{-1}$ ) for $45 \mathrm{~min}$ followed by stimulation with psoriasin or the positive control interleukin (IL)-17 (50 ng mL ${ }^{-1}$ ) for 18 h. (f) Psoriasin and IL-17 induced HMVEC-d migration. The (g) PI3K-inhibitor, LY204002, and the (h) nuclear factor kappa B inhibitor, CAPE, prevented psoriasin-induced migration. Neither LY294002 and CAPE alone nor dimethyl sulfoxide (used as a diluent control) had any effect on HMVEC-d migration. The photographs are representative examples of three individual experiments. Bar graphs display the mean $\pm \mathrm{SD}$ and the migration or total length of tube formation relative to the control. ns, nonsignificant.

Furthermore, we demonstrate that psoriasin regulates the expression of the psoriasis-associated angiogenic factors VEGF, HB-EGF, IL-8, MMP-1 and MMP-9 in $\mathrm{H}_{2} \mathrm{O}_{2}$-treated keratinocytes, as indicated by the diminished response to ROS when psoriasin is silenced with siRNA. We have previously shown that psoriasin in itself increases the intracellular levels of 
Fig 4. Psoriasin induces the expression of angiogenic factors in dermal endothelial cells. Human dermal-derived microvascular endothelial cells (HMVEC-d) were treated with psoriasin $\left(0 \cdot 15-1 \cdot 0 \mu \mathrm{g} \mathrm{mL}{ }^{-1}\right)$ for $24 \mathrm{~h}$. Expression was analysed by real-time quantitative polymerase chain reaction. Psoriasin treatment induced the mRNA expression of (a) vascular endothelial growth factor (VEGF; $\mathrm{n}=8$ ), (b) interleukin (IL)-8 $(\mathrm{n}=8)$, (c) matrix metalloproteinase (MMP)$1(\mathrm{n}=3)$ and (d) interleukin (IL)-6 ( $\mathrm{n}=8)$, while (e) reducing the mRNA expression of thrombospondin $1(n=6)$. (f) Furthermore, the expression of the psoriasin receptor RAGE (receptor for advanced glycation endproducts) was upregulated upon treatment $(\mathrm{n}=6)$. (g) Treatment with psoriasin also caused the production of IL-8, measured by enzymelinked immunosorbent assay of cell supernatant $(\mathrm{n}=7)$. VEGF $\left(10 \mathrm{ng} \mathrm{mL} \mathrm{m}^{-1}\right)$ was used as the positive control. The results are shown as the relative expression of treated cells in relation to untreated cells. The mRNA expression is normalized to GAPDH as the internal control. Data are expressed as mean \pm SD. $* \mathrm{P}<0.05, * * \mathrm{P}<0.01 . \mathrm{ns}$, nonsignificant.

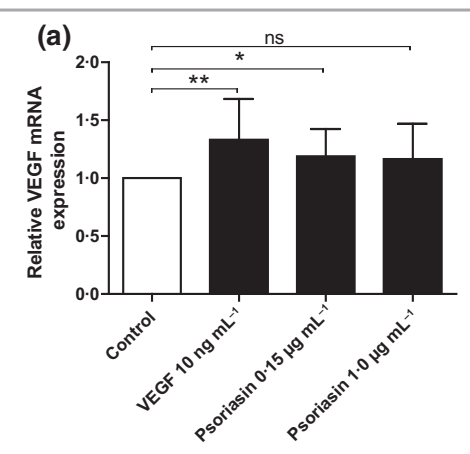

(c)
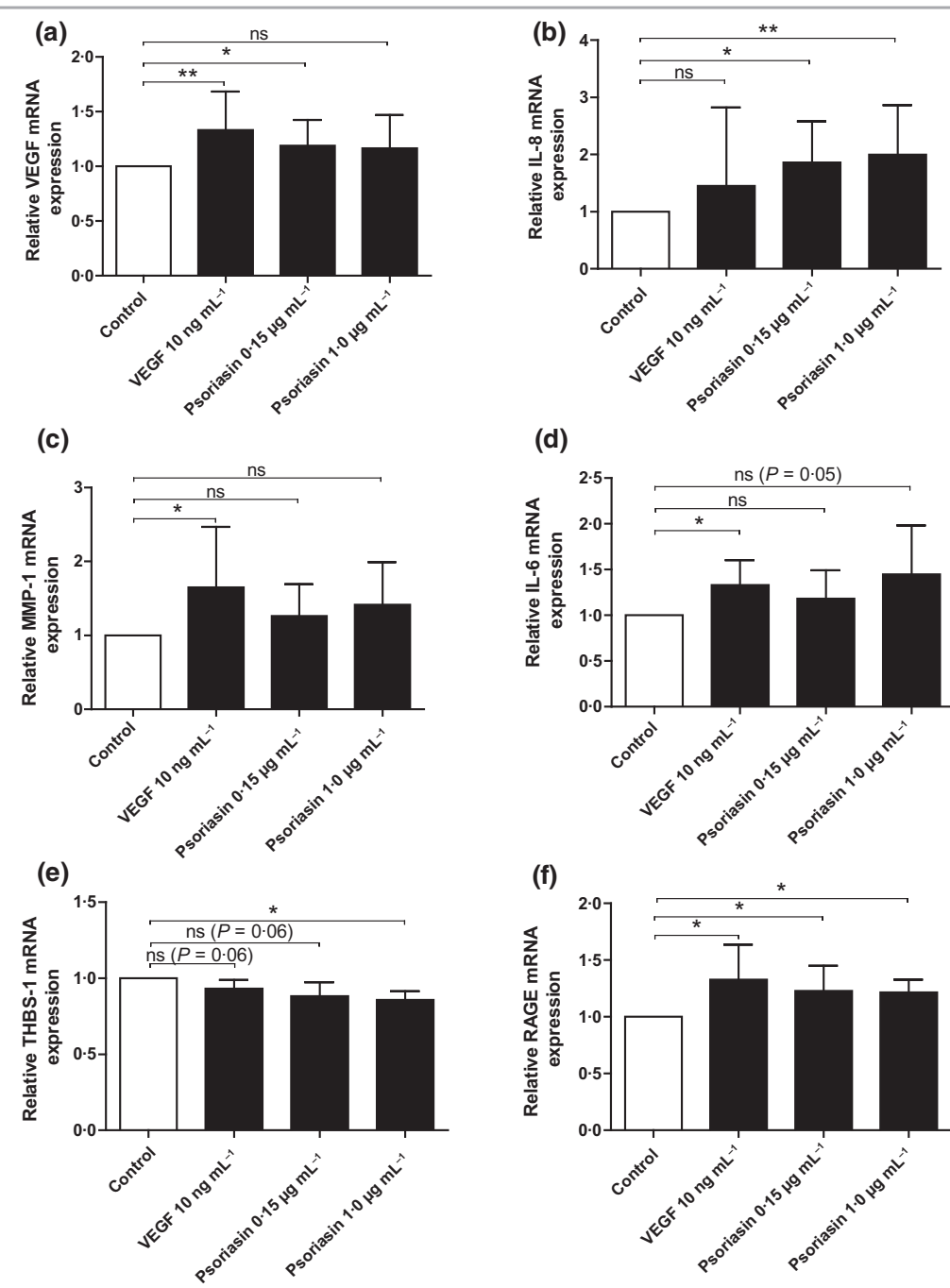

(d)

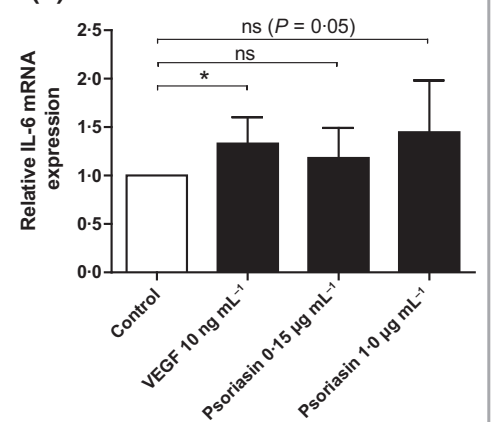

(f)

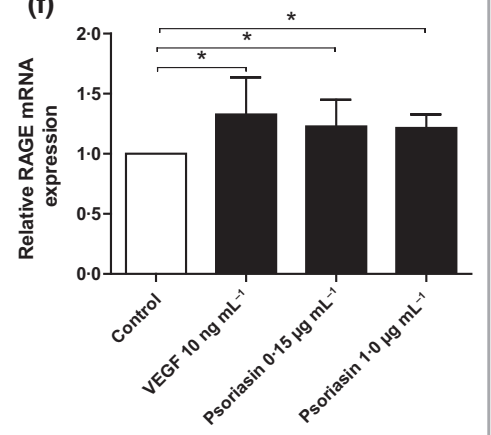

(g)

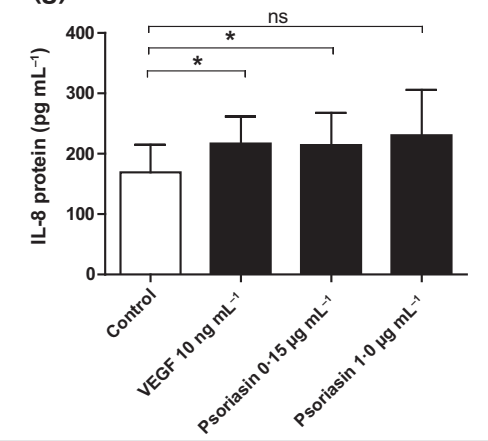

ROS in keratinocytes, suggesting a feedback loop where psoriasin is both induced by ROS and amplifies the angiogenic response to ROS. ${ }^{29}$

Among the factors that were regulated by psoriasin, VEGF is of particular interest because of its overexpression in psoriatic keratinocytes and its potent mitogenic effect on endothelial cells. ${ }^{6,40}$ Extracellular psoriasin also induced the expression of VEGF and IL-8 in dermal endothelial cells, in parallel with an induced cell proliferation. Furthermore, extracellular psoriasin also caused an increase in the expression of the RAGE receptor. As psoriasin is known to be a ligand of RAGE, ${ }^{41}$ this suggests that the upregulation of this receptor upon psoriasin treatment may potentiate the response to psoriasin in an autocrine fashion.

The regulation of MMPs by psoriasin is also of interest as they are involved in the proteolysis of the basement membrane, which is a critical step during the very early stages of angiogenesis. ${ }^{42}$ Similarly, HB-EGF, which is upregulated in the psoriatic lesion, ${ }^{43}$ is a critical molecular component in wound healing and angiogenesis. ${ }^{44}$ The increase in THBS-1 expression in 
$\mathrm{H}_{2} \mathrm{O}_{2}$-treated keratinocytes with silenced psoriasin expression is in line with previous findings where we have observed a negative correlation between psoriasin and THBS-1 in normal and ductal carcinoma in situ specimens. ${ }^{45}$ The microarray analysis of keratinocytes overexpressing psoriasin also suggests that psoriasin affects a broad range of angiogenic factors. We found that psoriasin is highly secreted and leads to the proliferation, migration and differentiation of dermal endothelial cells.

In conclusion, our findings demonstrate that psoriasin amplifies the stress-induced angiogenic response in keratinocytes and endothelial cells and is involved in potent proangiogenic activity in several crucial steps in the angiogenic process in vitro. These findings provide a link between psoriasin and angiogenesis, and suggest that the high expression of psoriasin contributes to the increased vascularization in psoriatic epidermis and the progression of the disease. Our findings raise the possibility that psoriasin could be evaluated as a novel antiangiogenic target in psoriasis.

\section{References}

1 Schon MP, Boehncke WH. Psoriasis. N Engl J Med 2005; 352:1899912.

2 Petzelbauer P, Pober JS, Keh A et al. Inducibility and expression of microvascular endothelial adhesion molecules in lesional, perilesional, and uninvolved skin of psoriatic patients. J Invest Dermatol 1994; 103:300-5.

3 Bacharach-Buhles M, el Gammal S, Panz B et al. The pseudo-elongation of capillaries in psoriatic plaques. Acta Derm Venereol Suppl (Stockh) 1994; 186:133-7.

4 Braverman IM, Yen A. Ultrastructure of the human dermal microcirculation. II. The capillary loops of the dermal papillae. J Invest Dermatol 1977; 68:44-52.

5 Creamer D, Sullivan D, Bicknell R et al. Angiogenesis in psoriasis. Angiogenesis 2002; 5:231-6.

6 Heidenreich R, Rocken M, Ghoreschi K. Angiogenesis drives psoriasis pathogenesis. Int J Exp Pathol 2009; 90:232-48.

7 Barton SP, Abdullah MS, Marks R. Quantification of microvascular changes in the skin in patients with psoriasis. Br J Dermatol 1992; 126:569-74

8 Micali G, Lacarrubba F, Musumeci ML et al. Cutaneous vascular patterns in psoriasis. Int J Dermatol 2010; 49:249-56.

9 Detmar M, Brown LF, Claffey KP et al. Overexpression of vascular permeability factor/vascular endothelial growth factor and its receptors in psoriasis. J Exp Med 1994; 180:1141-6.

10 Armstrong AW, Voyles SV, Armstrong EJ et al. Angiogenesis and oxidative stress: common mechanisms linking psoriasis with atherosclerosis. J Dermatol Sci 2011; 63:1-9.

11 Henno A, Blacher S, Lambert C et al. Altered expression of angiogenesis and lymphangiogenesis markers in the uninvolved skin of plaque-type psoriasis. Br J Dermatol 2009; 160:581-90.

12 Detmar M, Brown LF, Schon MP et al. Increased microvascular density and enhanced leukocyte rolling and adhesion in the skin of VEGF transgenic mice. J Invest Dermatol 1998; 111:1-6.

13 Ryan C, Korman NJ, Gelfand JM et al. Research gaps in psoriasis: opportunities for future studies. J Am Acad Dermatol 2014; 70:14667.

14 Madsen P, Rasmussen HH, Leffers $\mathrm{H}$ et al. Molecular cloning, occurrence, and expression of a novel partially secreted protein "psoriasin" that is highly up-regulated in psoriatic skin. J Invest Dermatol 1991; 97:701-12.
15 Donato R. Functional roles of S100 proteins, calcium-binding proteins of the EF-hand type. Biochim Biophys Acta 1999; 1450:191-231.

16 Hoffmann HJ, Olsen E, Etzerodt M, et al. Psoriasin binds calcium and is upregulated by calcium to levels that resemble those observed in normal skin. J Invest Dermatol 1994; 103:370-5.

17 Alowami S, Qing G, Emberley E et al. Psoriasin (S100A7) expression is altered during skin tumorigenesis. BMC Dermatol 2003; 3:1.

18 Ostergaard M, Wolf H, Orntoft TF et al. Psoriasin (S100A7): a putative urinary marker for the follow-up of patients with bladder squamous cell carcinomas. Electrophoresis 1999; 20:349-54.

19 Enerback C, Porter DA, Seth P et al. Psoriasin expression in mammary epithelial cells in vitro and in vivo. Cancer Res 2002; 62:43-7.

20 Matsumoto T, Murao S, Kito K et al. Modulation of S-100 genes response to growth conditions in human epithelial tumor cells. Pathol Int 1997; 47:339-46.

21 Algermissen B, Sitzmann J, LeMotte P et al. Differential expression of CRABP II, psoriasin and cytokeratin 1 mRNA in human skin diseases. Arch Dermatol Res 1996; 288:426-30.

22 Lee KC, Eckert RL. S100A7 (Psoriasin)-mechanism of antibacterial action in wounds. J Invest Dermatol 2007; 127:945-57.

23 Glaser R, Harder J, Lange $\mathrm{H}$ et al. Antimicrobial psoriasin (S100A7) protects human skin from Escherichia coli infection. Nat Immunol 2005; 6:57-64.

24 Jinquan T, Vorum H, Larsen CG et al. Psoriasin: a novel chemotactic protein. J Invest Dermatol 1996; 107:5-10.

25 Liang SC, Tan XY, Luxenberg DP et al. Interleukin (IL)-22 and IL17 are coexpressed by Th17 cells and cooperatively enhance expression of antimicrobial peptides. J Exp Med 2006; 203:2271-9.

26 Martinsson H, Yhr M, Enerback C. Expression patterns of S100A7 (psoriasin) and S100A9 (calgranulin-B) in keratinocyte differentiation. Exp Dermatol 2005; 14:161-8.

27 Dickson MA, Hahn WC, Ino $\mathrm{Y}$ et al. Human keratinocytes that express hTERT and also bypass a p16(INK4a)-enforced mechanism that limits life span become immortal yet retain normal growth and differentiation characteristics. Mol Cell Biol 2000; 20:1436-47.

28 Krop I, Marz A, Carlsson $\mathrm{H}$ et al. A putative role for psoriasin in breast tumor progression. Cancer Res 2005; 65:11326-34.

29 Skliris GP, Lewis A, Emberley E et al. Estrogen receptor-beta regulates psoriasin (S100A7) in human breast cancer. Breast Cancer Res Treat 2007; 104:75-85.

30 Sato $\mathrm{T}, \mathrm{Wu} \mathrm{X}$, Shimogaito $\mathrm{N}$ et al. Effects of high-AGE beverage on RAGE and VEGF expressions in the liver and kidneys. Eur $J$ Nutr 2009; 48:6-11.

31 Doroudi R, Andersson M, Svensson PA et al. Methodological studies of multiple reference genes as endogenous controls in vascular gene expression studies. Endothelium 2005; 12:215-23.

32 Shubbar E, Vegfors J, Carlstrom M et al. Psoriasin (S100A7) increases the expression of ROS and VEGF and acts through RAGE to promote endothelial cell proliferation. Breast Cancer Res Treat 2012; 134:71-8.

33 Rashmi R, Rao KS, Basavaraj KH. A comprehensive review of biomarkers in psoriasis. Clin Exp Dermatol 2009; 34:658-63.

34 Guzy RD, Hoyos B, Robin E et al. Mitochondrial complex III is required for hypoxia-induced ROS production and cellular oxygen sensing. Cell Metab 2005; 1:401-8.

35 Ioannou M, Sourli F, Mylonis I et al. Increased HIF-1 alpha immunostaining in psoriasis compared to psoriasiform dermatitides. J Cutan Pathol 2009; 36:1255-61.

36 Toker A, Cantley LC. Signalling through the lipid products of phosphoinositide-3-OH kinase. Nature 1997; 387:673-6.

37 Yao Z, Fanslow WC, Seldin MF et al. Herpesvirus Saimiri encodes a new cytokine, IL-17, which binds to a novel cytokine receptor. Immunity 1995; 3:811-21. 
38 Kunsch C, Medford RM. Oxidative stress as a regulator of gene expression in the vasculature. Circ Res 1999; 85:753-66.

39 Carlsson H, Yhr M, Petersson S et al. Psoriasin (S100A7) and calgranulin-B (S100A9) induction is dependent on reactive oxygen species and is downregulated by Bcl-2 and antioxidants. Cancer Biol Ther 2005; 4:998-1005.

40 Man XY, Yang XH, Cai SQ et al. Overexpression of vascular endothelial growth factor (VEGF) receptors on keratinocytes in psoriasis: regulated by calcium independent of VEGF. J Cell Mol Med 2008; 12:649-60.

41 Wolf R, Howard OM, Dong HF et al. Chemotactic activity of S100A7 (psoriasin) is mediated by the receptor for advanced glycation end products and potentiates inflammation with highly homologous but functionally distinct S100A15. J Immunol 2008; 181:1499-506.

42 Bhushan M, Young HS, Brenchley PE et al. Recent advances in cutaneous angiogenesis. Br J Dermatol 2002; 147:418-25.

43 Stoll SW, Elder JT. Retinoid regulation of heparin-binding EGF-like growth factor gene expression in human keratinocytes and skin. Exp Dermatol 1998; 7:391-7.
44 Stoll S, Garner W, Elder J. Heparin-binding ligands mediate autocrine epidermal growth factor receptor activation In skin organ culture. J Clin Invest 1997; 100:1271-81.

45 Petersson S, Shubbar E, Yhr M et al. Loss of ICAM-1 signaling induces psoriasin (S100A7) and MUC1 in mammary epithelial cells. Breast Cancer Res Treat 2011; 125:13-25.

\section{Supporting Information}

Additional Supporting Information may be found in the online version of this article at the publisher's website:

Fig S1. The overexpression of psoriasin induces protein secretion and the regulation of angiogenic mediators.

Table S1. Primer sequences used for quantitative real-time polymerase chain reaction.

Table S2. Regulators of angiogenesis in keratinocytes overexpressing psoriasin. 